\title{
OFFICE BUILDING OCCUPANCY MONITORING THROUGH IMAGE RECOGNITION SENSORS
}

\author{
MANNINO ANTONINO ${ }^{1}$, MORETTI NICOLA $^{1}$, DEJACO MARIO CLAUDIO $^{1}$, BARESI LUCIANO $^{2} \&$ \\ RE CECCONI FULVIO ${ }^{1}$ \\ ${ }^{1}$ Politecnico di Milano, ABC Department. \\ ${ }^{2}$ Politecnico di Milano DEIB Department.
}

\begin{abstract}
In the Architecture, Engineering, Construction and Operations (AECO) there is a growing interest in the use of the Building Information Modelling (BIM). Through integration of information and processes in a digital model, BIM can optimise resources along the lifecycle of a physical asset. Despite the potential savings are much higher in the operational phase, BIM is nowadays mostly used in design and construction stages and there are still many barriers hindering its implementation in Facility Management (FM). Its scarce integration with live data, i.e. data that changes at high frequency, can be considered one of its major limitations in FM. The aim of this research is to overcome this limit and prove that buildings or infrastructures operations can benefit from a digital model updated with live data. The scope of the research concerns the optimisation of FM operations. The optimisation of operations can be further enhanced by the use of maintenance smart contracts allowing a better integration between users' behaviour and maintenance implementation. In this case study research, the Image Recognition (ImR), a type of Artificial Intelligence (AI), has been used to detect users' movements in an office building, providing real time occupancy data. This data has been stored in a BIM model, employed as single reliable source of information for FM. This integration can enhance maintenance management contracts if the BIM model is coupled with a smart contract. Far from being a comprehensive case study, this research demonstrates how the transition from BIM to the Asset Information Model (AIM) and, finally, to the Digital Twin (i.e. a near-real-time digital clone of a physical asset, of its conditions and processes) is desirable because of the outstanding benefits that have already been measured in other industrial sectors by applying the principles of Industry 4.0.

Keywords: Building Information Modeling, Facility Management, Image recognition, smart contracts.
\end{abstract}

\section{INTRODUCTION}

The construction industry, compared to other sectors, presents a low level of digitisation [1]. However, there is a growing interest in using new technologies in order to improve building management and maintenance and reduce whole life cycle costs. Building Information Modelling (BIM) and the information management processes encompassed by ISO 19650-2 [2] are taking great advantages by the use of these technologies. However, BIM is used mainly for design and construction phases [3], despite the use phase represents the largest portion of the whole Life Cycle Cost (LCC) of the building process, from $65 \%$ up to $85 \%$ [2]-[3]. Among these costs, the ones related to energy consumption have a key-role in construction industry. In the European Union (EU) buildings account for $40 \%$ of total energy consumption, which is bound to increase [4]. Recent studies assert that a reliable occupancy detection can improve buildings energy management and savings [3], [5]-[9]. According to Christensen et al. [10] occupancy information can be used to control systems and equipments, which can be operated according to the number of occupants in the building, reducing the energy consumption. Harle [11] proved that occupancy detection can help saving up to $50 \%$ in lighting costs. Moreover, occupancy detection can reduce Heating, Ventilation and Air Conditioning (HVAC) energy consumption up to 20\% [12].

Several types of sensors can be used to gather occupancy data from a building thanks to their widespread availability and ease of installation. Occupancy detection in buildings is 
often carried out through Passive InfraRed (PIR) and ultrasonic sensors [13]. Some applications of PIR sensors to control lighting in internal spaces can be found in literature [14], but this kind of sensor is not suitable for occupancy detection in large internal spaces (e.g. offices) [9], [10]. Ultrasonic sensors were introduced for demand-driven applications as an improvement over PIR sensors as they do not require line of sight and continuous movement [15].

Although sensors-networks have a great potential to gather reliable information on occupancy, sometimes, especially in existing buildings, their use is hindered by high installation costs. Hence, one of the constrains of the research was to use inexpensive but reliable sensors.

Among all the types of sensors systems used for occupancy detection, image detection systems are the only ones able to provide information on location, count, activity, identity and track at the same time [15]. Despite an expensive and specific hardware is required by an image detection system to be able to provide comprehensive information on a whole building, a single camera can often cover a wide floor area and, consequently, the whole system cost may be even lower than the one based on an ultrasonic sensors' network. Not to mention that a system based on ultrasonic sensors is not able to provide all the information that can be gathered by an image detection system.

In this paper we propose a method to measure precise real-time occupancy data in crowded environment detecting people movements using Image Recognition (ImR) sensors and computer vision. This method is part of a wider research aiming at improving Facility Management (FM) using BIM as a single source of information. The aim of this research is to prove that a BIM model fed with real time data coupled with a smart contract can change FM current practice. In order to gradually address the wider research objective, the method presented through this article has been validated on a case study building, an office building in Milano.

\section{BACKGROUND}

Large amount of data, which come from buildings, need to be managed. To date, there are several tools and methodologies to manage the built environment: two of the most common are Computer Aided Facility Management (CAFM) and the Computerized Maintenance Management Systems (CMMS) [16]. On the other hand, the BIM approach allows also the integration of information for buildings maintenance and management. The integration between real-time information and BIM fosters the development of a single source information also known as Digital Twin, which could improve decision making, energy and asset management [17]. To define occupancy in a space and update real-time data in the digital model two types of detection systems can be used: individualized and non-individualized, based on whether or not an occupant can be identified [18]. Among non-individualized systems can be encompassed passive sensors such as infrared sensors, $\mathrm{CO}_{2}$ sensors, light sensors or ultrasonic sensors, which provide data that can be used to calculate the occupancy indirectly. Even if these kinds of sensors do not present any privacy issues because they cannot identify users; they could present some problems in counting people, especially in a crowded environment [19]. Moreover, also environmental conditions might limit sensors' usability [19].

Individualized detection systems use instead images, radio signals, Radio-Frequency IDentification (RFID) tags which are more reliable in counting people [20] even if they can present some privacy issues due to people personal data collection.

In the last years several of the above-mentioned types of detection systems were used to detect and count people in building spaces [21]. Each method present advantages and limitations (e.g. PIR sensors cannot detect steady people).

Therefore, Benezeth et al. [22] proposed a system to count people using an analysis based on camera and video. The system they propose is based on three steps: 1) change detection 
using a background model updated at different levels to manage the most common variations of the environment 2) objects tracking 3) recognition. Despite privacy issue, their system gives a detection rate of $97 \%$.

To overcome privacy problems, Sarkar et al. [23] used an image sensor, without storage capacity, for daylight harvesting and occupancy detection. However, with this kind of system is possible only to detect whether or not the room is occupied.

\section{METHODOLOGY}

The proposed occupancy detection system aims at updating a digital asset with live data. The purpose is to provide improved FM services. This system is based on the use of the following components: an Image Recognition sensor integrated with a Raspberry board and connected with a database on a server which, through a wireless connection, updates the BIM model of the building (Fig. 1).

To achieve an increased level of data reliability and an automation in information storage and management, an Artificial Intelligence (AI) installed on the Raspberry board is coupled with the image sensor. In this way a better recognition both of people and their movements is possible, and data are automatically stored in the digital twin of the building.

The camera is installed on the false ceiling in order to better recognize people and to avoid privacy issues. When a person, or more than one, enter in the field of view of the camera, the Artificial Intelligence on the Raspberry analyses the frames in order to detect and track movements and direction of all people in the captured images. Frames are divided in four parts (Fig. 2), one for each possible direction people can take.

To each part is assigned a certain room in the floor of the building. When a person crosses two of the four sectors composing the frame (the red circle in Fig. 2), the script updates the database on the server adding (+1) to the occupancy value of the room assigned to the last sector occupied by the person and subtracting $(-1)$ from the occupancy value of the room assigned to the sector he/she left.

Data are stored in the digital model, which allows to trigger maintenance work orders when the threshold defined in the maintenance plan is reached.

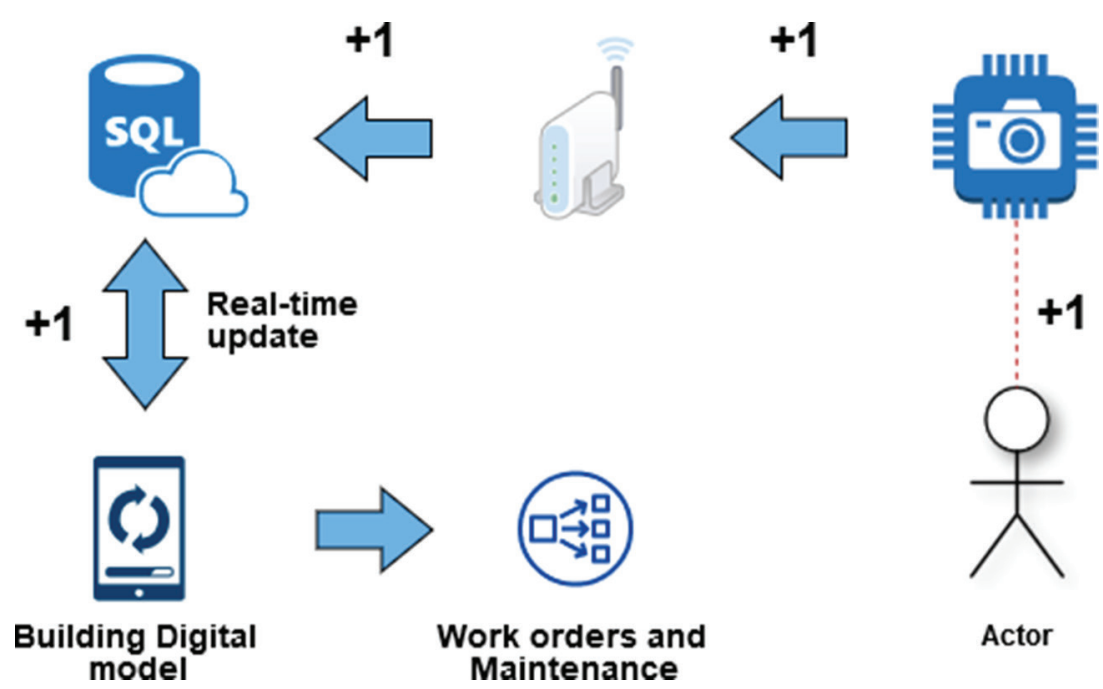

Figure 1: Occupancy detection components and connections. 


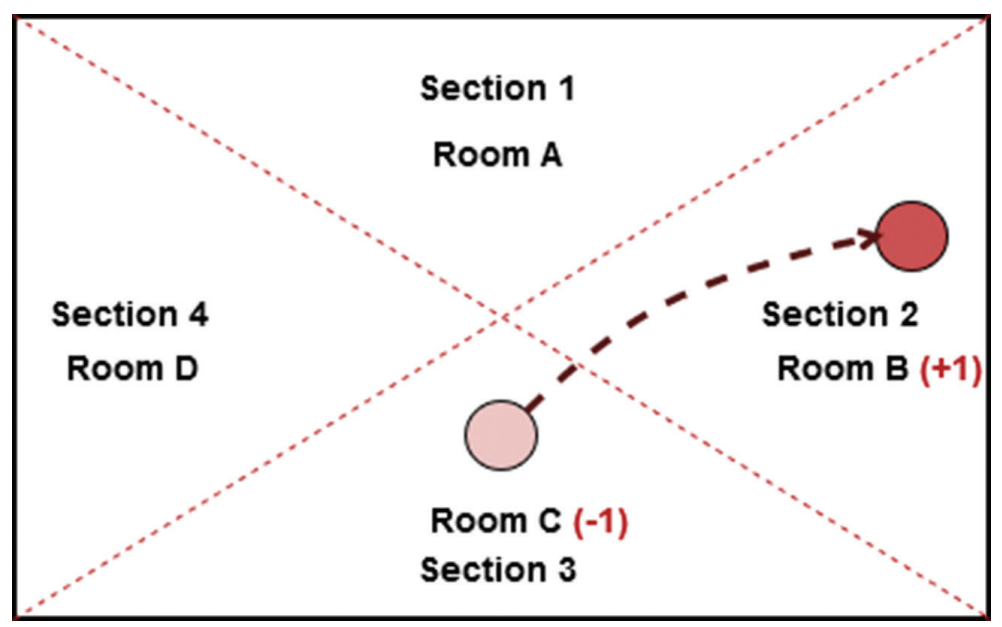

Figure 2: The four sections of the frame.

\section{SIMULATION AND VALIDATION}

Real-time data plays a key role in buildings management and maintenance, contributing to the improvement of the whole life cycle cost.

In this article we evaluate an image recognition method able to better define occupancy in building spaces. To investigate the accuracy of the proposed system in collecting occupancy information, a test in an office building of Politecnico di Milano has been carried out. To perform this test, a Raspberry Pi single-board computer (Fig. 3a) and a camera (Fig. 3b) have been employed. These devices are connected to a wi-fi network.

An Artificial Intelligence which read and analyse frames of the camera and recognize people and their directions has been loaded on the Raspberry board. The BIM model was realized with Autodesk Revit and updated with real-time data using Revit DB Link. As cloud database was used Microsoft Azure SQL Database.

For this simulation, the camera was installed on the false ceiling (height $=240 \mathrm{~cm}$ ) at the intersection of two corridors. Detected area was about 200 x $160 \mathrm{~cm}$ divided in four parts by
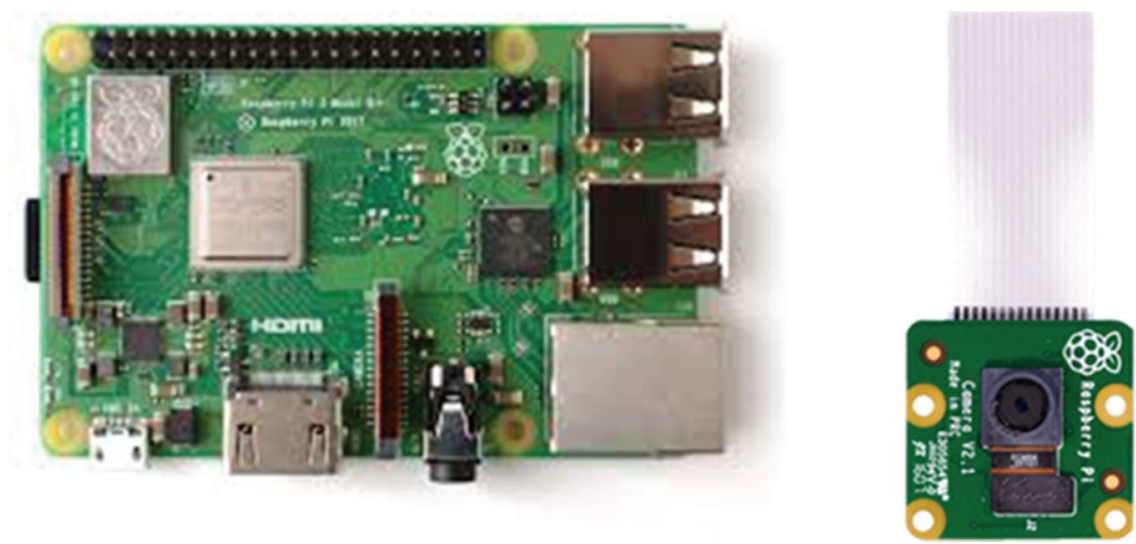

Figure 3: (a) Raspberry Pi board (b) camera. 
two virtual lines defined by the AI. In order to assign occupancy values to the building spaces, the four parts of the frame were assigned to four different spaces defined in the virtual model (hence also in the SQL database).

In order to evaluate the system accuracy, gathered data have been compared and validated on site by the authors registering people passing under the camera and their directions. Data collected were stored also with information about time and room identifier.

The SQL database in which occupancy data was stored coupled with the BIM model define the digital model of the building, which is always updated with real-time data. Both Realtime data and historical data were stored in the database, and this information can be always obtained by the maintenance services provider.

\section{RESULTS AND DISCUSSION}

A first problem emerging from this occupancy detection system is the privacy issues due to the sensors with the camera in a public workplace. To overcome this drawback, the camera has been placed on the top, installed to the false ceiling and oriented vertically towards the floor. No faces have been detected and the script uploaded on the Raspberry board cannot recognize people. Moreover, images have not been stored and, to reduce noises and improve the counting of people, have been modified through morphological operations, which makes impossible to recognize people (Fig. 4).

If the data are owned by the employer and not by the maintenance service provider, additional privacy issues could rise (e.g. occupancy value of workers' personal offices). In this case, to overcome this problem, after the maintenance work order is sent to the maintenance provider, just the average value of floor occupancy could be stored in the digital model. In this way major privacy issues could be avoided.

The script used in this simulation allows to detect moving objects of a certain size which are recognized as persons, tracked and added to the occupancy value of the space towards which they are moving.

If on the one hand this occupancy detection system is more accurate than others (e.g. ultrasonic sensors), on the other hand some difficulties arised when more than two people enter in the field of view of the camera. The person tracking should be improved to better count people in a crowded environment.

All data collected can be used not only for a technical control proposal but also to define a smart contract associated to a maintenance plan. Real-time data about the flow of people passing through a monitored area are useful in order to define a smart contract which

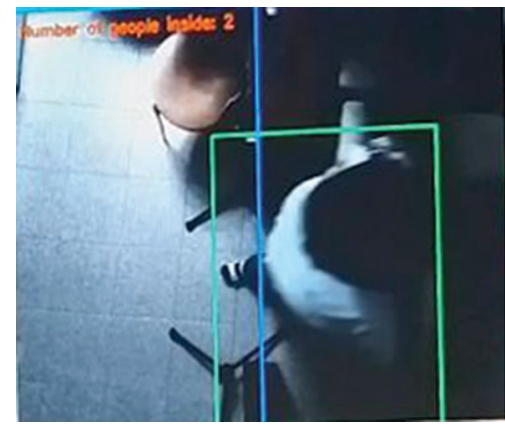

Figure 4: Frame from camera before (a) and after (b) morphological operations.

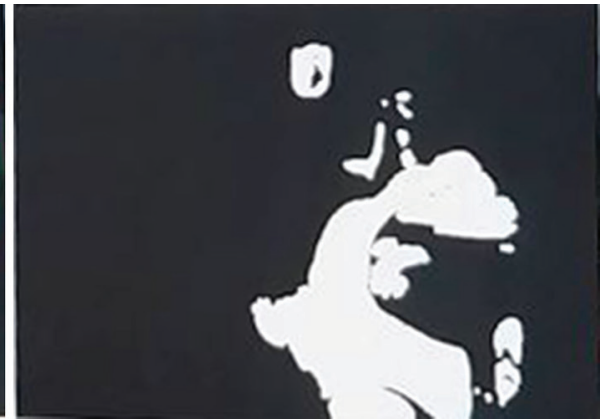


guarantees an optimized maintenance service based both on planned maintenance and occupancy information.

The real-time occupancy control of building spaces in this case is considered as the trigger of the smart contract, which is activated when a predefined threshold is reached and allows automated transaction among stakeholders [24].

With real-time occupancy data is possible to improve also emergency management. Querying the Digital Twin of the building and knowing in real time how many people are inside the building and where they are located, could improve emergency operations by reducing intervention times or planning the optimal path for rescuers.

\subsection{Error evaluation}

The reliability of the data collected in this simulation with the image sensor was tested. System precision and accuracy in people detection has been calculated using (1).

$$
\text { error }=\frac{|\mathrm{N} 1-\mathrm{N} 2|}{\mathrm{N} 2} \times 100 \% .
$$

$\mathrm{N} 1=$ People counted by image sensor

N2 = People counted by authors.

Detection rate of this system is about $71 \%$. Most of the wrong detections are due to people crossing the monitored area too quickly. Furthermore, in other cases the error is caused by people stopping under the sensor: they have been counted in more than a space and wrong occupancy value has been assigned to involved offices. However, the main limitation of this system is to detect and track more than two people crossing at the same time the monitored area: $80 \%$ of crossings with more than two persons have not been counted correctly. On the other hand, considering the crossing of a single person in the monitored area, detection rate becomes $79 \%$.

\subsection{Costs evaluation and savings}

With data collected in the simulation, also a cost analysis has been carried out based on the planned cleaning service and the savings provided by the implementation of the proposed approach. The number of sensors needed to create the network to cover the whole building floor was estimated according to Figure 5.

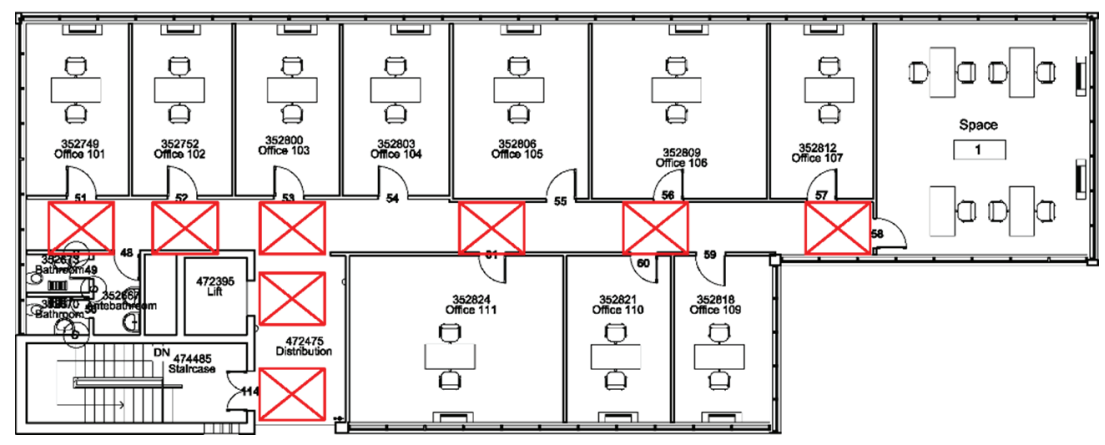

Figure 5: Number of sensors necessary to create a network. 
Eight sensors are needed to monitor offices' occupancy in the wing of the considered building floor. The number of sensors has been calculated considering the area covered by the field of view of the camera. The costs estimated are summarized in Table 1.

Cleaning interventions are planned three times a week in all the offices. Since the offices are not always occupied during the week, the number of cleaning interventions could be reduced. Considering occupancy data collected during the simulation, savings have been calculated. Occupancy data in all offices have been manually collected by authors. The Raspberry board has been used to calculate system's accuracy. For all offices, occupancy information have been used to calculate the number of cleaning interventions in a week. Cleaning of bathroom and corridors are not considered in this study since these spaces are daily cleaned. Data have been collected in two weeks (from Monday to Friday) from 9:00 to 19:00. Considering a cleaning cost of $0.35 € / \mathrm{m}^{2}$, the monthly costs for the planned cleaning services is $1050.00 €\left(0.35 € / \mathrm{m}^{2} \times 250 \mathrm{~m}^{2} \times 12\right.$ times in a month). Occupancy-based cleaning service is activated when a threshold of 16 hours is reached. Tables 2 and 3 summarize occupancy data measured, cleaning costs and savings for the first and the second week.

Table 1: Sensors network costs.

\begin{tabular}{lccc}
\hline Services / devices & Unit cost & Quantity & Total \\
\hline Raspberry Pi Board & $42.14 €$ & 8 & $337.12 €$ \\
Raspberry Camera & $24.99 €$ & 8 & $199.92 €$ \\
Installation Costs & $50.00 €$ & 8 & $400.00 €$ \\
Software development & $2500.00 €$ & 1 & $2500.00 €$ \\
Total investment & & & $3437.00 €$ \\
\hline
\end{tabular}

Table 2: Occupancy and cleaning cost. Savings: $45 \%$ of planned costs (1st week).

\begin{tabular}{cccccccc}
\hline Office & $\mathbf{m}^{2}$ & $\begin{array}{c}\text { Occupancy } \\
\text { measured (h) }\end{array}$ & \multicolumn{2}{c}{ C.S. } & \multicolumn{2}{c}{ Costs $(\boldsymbol{\epsilon})$} & Savings (€) \\
\hline & & & $\mathbf{P}$ & $\mathbf{R}$ & $\mathbf{P}$ & $\mathbf{R}$ & \\
101 & 17 & 5 & 3 & 1 & 17.85 & 5.95 & 11.90 \\
102 & 16.5 & 22 & 3 & 1 & 17.30 & 5.80 & 11.50 \\
103 & 17 & 4 & 3 & 1 & 17.85 & 5.95 & 11.90 \\
104 & 17.5 & 7 & 3 & 1 & 18.40 & 6.15 & 12.25 \\
105 & 23 & 18 & 3 & 1 & 24.15 & 8.05 & 16.10 \\
106 & 29 & 27 & 3 & 1 & 30.45 & 10.15 & 20.30 \\
107 & 17 & 24 & 3 & 1 & 17.85 & 5.95 & 11.90 \\
Space & 47 & 50 & 3 & 3 & 49.35 & 49.35 & 0 \\
109 & 15 & 26 & 3 & 1 & 15.75 & 5.25 & 10.50 \\
110 & 16.5 & 23 & 3 & 1 & 17.30 & 5.80 & 11.50 \\
111 & 34 & 48 & 3 & 3 & 35.70 & 35.70 & 0 \\
Total & 249.5 & $/$ & 3 & $/$ & 262.00 & 144.10 & 117.90 \\
\hline
\end{tabular}


Table 3: Occupancy and cleaning cost. Savings: $41 \%$ of planned costs (2nd week).

\begin{tabular}{cccccccc}
\hline Office & $\mathbf{m}^{2}$ & $\begin{array}{c}\text { Occupancy } \\
\text { measured }(\mathbf{h})\end{array}$ & \multicolumn{2}{c}{ C.S. } & \multicolumn{2}{c}{ Costs $(\boldsymbol{€})$} & $\begin{array}{c}\text { Savings } \\
(\boldsymbol{\epsilon})\end{array}$ \\
\hline & & & $\mathbf{P}$ & $\mathbf{R}$ & $\mathbf{P}$ & $\mathbf{R}$ & \\
101 & 17 & 14 & 3 & 1 & 17.85 & 5.95 & 11.90 \\
102 & 16.5 & 16 & 3 & 1 & 17.30 & 5.80 & 11.50 \\
103 & 17 & 32 & 3 & 2 & 17.85 & 11.90 & 5.95 \\
104 & 17.5 & 17 & 3 & 1 & 18.40 & 6.15 & 12.25 \\
105 & 23 & 22 & 3 & 1 & 24.15 & 8.05 & 16.10 \\
106 & 29 & 27 & 3 & 1 & 30.45 & 10.15 & 20.30 \\
107 & 17 & 27 & 3 & 1 & 17.85 & 5.95 & 11.90 \\
Space & 47 & 50 & 3 & 3 & 49.35 & 49.35 & 0 \\
109 & 15 & 41 & 3 & 2 & 15.75 & 10.50 & 5.25 \\
110 & 16.5 & 19 & 3 & 1 & 17.30 & 5.80 & 11.50 \\
111 & 34 & 49 & 3 & 3 & 35.70 & 35.70 & 0 \\
Total & 249.5 & $/$ & 3 & $/$ & 262.00 & 155.30 & 106.70 \\
\hline
\end{tabular}

C.S. $=$ Cleaning services $P=$ Planned $R=$ Real

Data collected show how, in a period in which not all offices are occupied for the entire working day, is possible to save up to $40 \%$ of planned costs ( $45 \%$ the first week and $41 \%$ the second week). In most cases, cleaning services, should be provided just once a week. Finally, considering a saving of $40 \%$ on the planned cleaning cost in a month, the initial investment could be recovered in about 8 months.

\section{CONCLUSIONS}

Despite is not possible to consider the update of the digital model as an automated process, is possible to consider the proposed approach as a further step towards the creation of a Digital Twin. No issues were found connecting real-time data collected through image sensor with the BIM model. Furthermore, both real-time data and historical data on room occupancy become a great value for building management and have the potential to optimize maintenance and services.

To overcome privacy issue sensors have been installed on the false ceiling and tilted towards the floor, no images are stored and the AI on the Raspberry board applies filters that optimize the counting of people but not their recognition. Moreover, two different scenarios can be considered for addressing the privacy issues: in the first scenario data are owned by maintenance services provider and, through the definition of contractual clauses on the confidentiality of data used to provide services, is possible to guarantee the privacy of the occupants. In the second scenario data are owned by the employer; in this case, to overcome all privacy issues, after the maintenance work order execution it is possible to save data history either guaranteeing the anonymity of data or considering an average of the occupancy value for each floor. 
Moreover, occupancy real-time data can also improve emergency management revealing both which rooms/spaces are still occupied and the best path for the rescuers.

The proposed sensor system can highly increase maintenance optimization, despite it needs further implementation. Triggering maintenance operations not only by frequencies defined in the maintenance plan, but also using occupancy data can reduce costs during the life cycle of the building. In this research savings for cleaning services can be up to $45 \%$ in a single week. However, this kind of system will not always guarantee reliable measures due to its difficulties to recognize and track more than two people in the monitored area and still need further improvements.

Despite the proposed approach still has to be tested in more case studies, a further automation in the FM services can be achieved. Hence, the next step concerns also a better automation in the update of the BIM model and the further development of the digital twin of the physical asset. Thanks to these data collected and managed through the Digital Twin, it will be possible to trigger maintenance interventions once settled Key Performance Indicator (KPI) thresholds, defined within the maintenance plan, are reached. Finally, with the integration of the maintenance plan in a smart contract for maintenance management, a better integration between users' behaviour and maintenance implementation is possible.

\section{ACKNOWLEDGEMENTS}

The authors would like to express their gratitude to Lorenzo Randazzo, Jorge Prieto Pereira, Gioele Pozzi, Luca Villa and Karolina Marianna Wadrzyk for their valuable contribution.

\section{REFERENCES}

[1] Manyika, J. et al., "Digital America: A tale of the haves and have-mores," 2015. [Online]. available: https://www.mckinsey.com/industries/high-tech/our-insights/digital-america-a-tale-of-the-haves-and-have-mores

[2] ISO 19650-2:2018, Organization and Digitization of Information About Buildings and Civil Engineering Works, Including Building Information Modelling (BIM)Information Management Using Building Information Modelling, 2018.

[3] Chung, S.W., Kwon, S.W., Moon, D.Y. \& Ko, T.K., Smart facility management systems utilizing open BIM and augmented/virtual reality. ISARC 2018-35th Int. Symp. Autom. Robot. Constr. Int. AEC/FM Hackathon Futur. Build. Things, 2018.

[4] 2010/31/EU, Directive 2010/31/EU of the european parliament and of the Council of 19 May 2010 on the Energy Performance of Buildings. pp. 13-35, 2010.

[5] Akanmu, A., Anumba, C. \& Messner, J., Critical review of approaches to integrating virtual models and the physical construction. International Journal of Construction Management, 14(4), pp. 267-282, 2014. https://doi.org/10.1080/15623599.2014.972021

[6] U.S. DoE, Buildings energy databook, 2011.

[7] Martinaitis, V., Zavadskas, E.K., Motuziene, V. \& Vilutiene, T., Importance of occupancy information when simulating energy demand of energy efficient house : A case study. Energyand Buildings, 101,pp. 64-75,2015.https://doi.org/10.1016/j.enbuild.2015.04.031

[8] Azar, E. \& Menassa, C.C., Evaluating the impact of extreme energy use behavior on occupancy interventions in commercial buildings. Energy and Buildings, 97, pp. 205-218, 2015. https://doi.org/10.1016/j.enbuild.2015.03.059

[9] Kleiminger, W., Mattern, F. \& Santini, S., Predicting household occupancy for smart heating control : A comparative performance analysis of state-of-the-art approaches. Energy and Buildings, 85, pp. 493-505, 2014. https://doi.org/10.1016/j.enbuild.2014.09.046 
[10] Christensen, K., Melfi, R., Nordman, B., Rosenblum, B. \& Viera, R., Using existing network infrastructure to estimate building occupancy and control plugged-in devices in user workspaces Bruce Nordman and Ben Rosenblum Raul Viera. International Journal of Communication Networks and Distributed Systems, 12(1), 2014. https://doi. org/10.1504/ijcnds.2014.057985

[11] Harle, R.K. \& Hopper, A., The potential for location-aware power management. in Proceedings of the 10th International Conference on Ubiquitous Computing, 2008, pp. 302-311.

[12] Erickson, V.L. \& Cerpa, A.E., Occupancy based demand response HVAC control strategy. In Proceedings of the 2nd ACM Workshop on Embedded Sensing Systems for Energy-Efficiency in Buildings, 2010, pp. 7-12.

[13] Dodier, R.H., Henze, G.P., Tiller, D.K. \& Guo, X., Building occupancy detection through sensor belief networks. Energy and Buildings, 38(9), pp. 1033-1043, 2006. https://doi.org/10.1016/j.enbuild.2005.12.001

[14] Von Neida, B., Maniccia, D. \& Tweed, A., An analysis of the energy and cost savings potential of occupancy sensors for commercial lighting systems an analysis of the energy and cost savings potential of occupancy sensors for commercial lighting systems. Journal of the Illuminating Engineering Society, 30(2), pp. 111-125, 2001. https://doi.org/10.1080/00994480.2001.10748357

[15] Labeodan, T., Zeiler, W., Boxem, G. \& Zhao, Y., Occupancy measurement in commercial office buildings for demand-driven control applications-A survey and detection system evaluation. Energy and Buildings, 93, pp. 303-314, 2015. https://doi. org/10.1016/j.enbuild.2015.02.028

[16] Volk, R., Stengel, J. \& Schultmann, F., Building information modeling (BIM) for existing buildings - Literature review and future needs. Automation in Construction, $\mathbf{4 3}$, p. 204, 2014.

[17] Centre for Digital Built Britain, The Gemini Principles, 2018.

[18] Li, N., Calis, G. \& Becerik-gerber, B., Measuring and monitoring occupancy with an RFID based system for demand-driven HVAC operations. Automation in Construction, 24, pp. 89-99, 2012. https://doi.org/10.1016/j.autcon.2012.02.013

[19] Kuutti, J., A Test Setup for Comparison of People Flow Sensors, pp. 1-89, 2012.

[20] Chen, J. Chen, H. \& Luo, X., Collecting building occupancy data of high resolution based on WiFi and BLE network. Automation in Construction, 102, pp. 183-194, 2019. https://doi.org/10.1016/j.autcon.2019.02.016

[21] Zhao, Y., Zeiler, W., Boxem, G. \& Labeodan, T., Virtual occupancy sensors for realtime occupancy information in buildings. Building and Environment, 93, pp. 9-20, 2015. https://doi.org/10.1016/j.buildenv.2015.06.019

[22] Benezeth, Y., Laurent, H., Emile, B. \& Rosenberger, C., Towards a sensor for detecting human presence and characterizing activity. Energy and Buildings, 43(2-3), pp. 305-314, 2011. https://doi.org/10.1016/j.enbuild.2010.09.014

[23] Sarkar, A., Fairchild, M. \& Salvaggio, C., Integrated daylight harvesting and occupancy detection using digital imaging. Sensors, Cameras, and Systems for Industrial/Scientific Applications IX, 6816, p. 68160F, 2008.

[24] Moretti, N. \& Re Cecconi, F., Blockchain application to maintenance smart contracts. In Research in Building Engineering - EXCO'18, 2018. 